\title{
Drug Reaction with Eosinophilia and Systemic Symptoms Associated with Cytomegalovirus
}

\section{Reactivation Leuco-encephalopathy}

\section{Rania Zouari $^{1,2}$ *, Ines Bedoui ${ }^{1,2,3}$, Hajer Derbali ${ }^{1,2}$, Najiba Fekih-Mrissa ${ }^{3,4}$, Malek Mansour ${ }^{1,2}$, Jamel Zaouali ${ }^{1,2}$, Ridha} Mrissa $^{1,2}$

${ }^{1}$ Department of Neurology, Military Hospital of Instruction of Tunis, Tunisia.

${ }^{2}$ Faculty of medicine of Tunis, Tunisia.

${ }^{3}$ Molecular Biology Unit (UR17DN06), Tunisia.

${ }^{4}$ Laboratory of Hematologie, Military Hospital of Instruction of Tunis, Tunisia.

*Corresponding Author: Rania Zouari, Department of Neurology, Military Hospital of Instruction of Tunis, Tunisia.

Received date: 18 October 2021; Accepted date: 30 October 2021; Published date: 05 November 2021

Citation: Zouari R, Bedoui I, Derbali H, Fekih-Mrissa N, Mansour M, et al. (2021) Drug Reaction with Eosinophilia and Systemic Symptoms Associated with Cytomegalovirus Reactivation Leuco-encephalopathy. J Med Case Rep Case Series 2(13): https://doi.org/10.38207/JMCRCS/2021/0213196

Copyright: (c) 2021 Rania Zouari. This is an open-access article distributed under the terms of the Creative Commons Attribution License, which permits unrestricted use, distribution, and reproduction in any medium, provided the original author and source are credited.

\begin{abstract}
Drug Reaction with Eosinophilia and Systemic Symptoms (DRESS) is a severe drug-induced cutaneous adverse reaction characterized by maculopapular rash, fever, lymphadenopathy, eosinophilia, and involvement of internal organs. DRESS has an unpredictable prognosis predominantly due to herpes viruses' reactivation. Here, we report the case of a 57-year-old female with a past history of cytomegalovirus (CMV) infection who developed DRESS two weeks following carbamazepine treatment onset with cytomegalovirus reactivation. She presented clinically with fever, extensive erythematous plaques, axillar adenopathy, and pneumonia associated with cognitive impairment and swallowing difficulties. Laboratory tests revealed lymphopenia and elevated C-reactive protein. A brain MRI showed bilateral, diffuse T2-weighted hyperintensities. Blood and cerebrospinal fluid CMV Polymerase Chain Reaction (PCR) was positive. The patient's condition progressively improved after carbamazepine withdrawal and ganciclovir treatment.
\end{abstract}

Keywords: DRESS - carbamazepine - cytomegalovirus - encephalopathy

\section{Introduction}

Drug Reaction with Eosinophilia and Systemic Symptoms (DRESS) is a severe, potentially life-threatening, delayed hypersensitivity reaction. Drugs that are most frequently implicated include aromatic antiepileptic drugs, allopurinol, and antibiotics [1]. Typical manifestations include skin eruptions, fevers, lymphadenopathy, eosinophilia, and reactive lymphocytosis. DRESS syndromeassociated reactivation of herpes viruses can frequently foster wide-

\section{Case report}

A 57-year-old woman, with no previous history of infection, was initially admitted to the emergency department complaining of acute-onset fever $\left(39^{\circ} \mathrm{C}\right)$ and a rapidly progressing headache. This presentation followed a treatment two weeks prior with carbamazepine for trigeminal neuralgia. A neurological examination was normal at that time. Her neck was supple, and she had no skin abnormalities. Laboratory examination revealed an elevated white blood cell count $(14000 / \mu \mathrm{L})$ with lymphopenia $(600 / \mu \mathrm{L})$ and an elevated C-reactive protein $(139 \mathrm{mg} / \mathrm{dL})$. A brain scan revealed no abnormalities. An analysis of cerebrospinal fluid ruled out central ranging internal organ involvement. Most commonly the liver, kidneys, lungs, and heart are affected [2]. However, central nervous system involvement is fairly uncommon.

We report the case of a 57-year-old woman who developed leucoencephalopathy due to carbamazepine-induced DRESS associated with cytomegalovirus reactivation to highlight this complication.

nervous system infection (WBC: $1 / \mathrm{mm}^{3}$ and proteins: $0.21 \mathrm{~g} / \mathrm{L}$ ). She was treated with cefixime and ofloxacin without a good outcome.

Two weeks later, the patient was referred to our Department of Neurology for a disorder of consciousness associated with persistent fever. On admission, blood pressure was $120 / 70 \mathrm{mmHg}$ with a pulse rate of 92 beats $/ \mathrm{min}$ and a body temperature of $38.5^{\circ} \mathrm{C}$. Her neurological examination revealed a deep-stupor mental state with confusion, bradykinesia, tremor, rigidity predominately affecting the right hand, swallowing difficulties, and pyramidal syndrome (a left hemiparesis, brisk reflexes, and extensor plantar responses). There was no neck stiffness, yet her eye fundus examination showed a 
papillary edema stage 4 . A physical examination revealed extensive erythematous itchy plaques over both lower limbs and the abdominal area, as well as axillary adenopathy. A blood workup showed lymphopenia $(700 / \mu \mathrm{L})$, elevated aspartate aminotransferase (AST) (59 UI/L), and alanine aminotransferase (ALT) (83 UI/L), as well as elevated CRP (117mg/dL). She had neither leukocytosis $(4200 / \mu \mathrm{L})$ nor eosinophilia $(200 / \mu \mathrm{L})$. Chest radiography demonstrated bilateral interstitial shadows within both lung fields suggestive of atypical pneumonitis. Brain magnetic resonance imaging (MRI) revealed bilateral, diffuse, and symmetrical T2-weighted and FLAIRweighted hyperintensities in cerebral and cerebellar white matter tracts sparing $U$ fibers and the corpus callosum. These abnormalities were also found within the thalamus nuclei and the brainstem (figure I). Spectroscopy showed a decreased N-Acetyl Aspartate (NAA) and a reduced N-Acetyl Aspartate/Choline ratio. A lumbar puncture found a clear CSF with normal protein $(0.22 \mathrm{~g} / \mathrm{L})$, a glucose level of $(2.7 \mathrm{mmol} / \mathrm{L})$, no leucocytes, no tumoral cells, and an undetectable herpes simplex virus (HSV) by polymerase chain reaction (PCR). Tests for anti-ANA, anti-DNA, anti-ENA, antiANCA, and anti-phospholipid antibodies were negative. Serological investigations were performed for several viruses and bacteria and revealed the absence of active infection of human immunodeficiency virus, hepatitis viruses, Epstein-Barr virus (EBV), HSV-1 and 2, varicella-zoster viruses $(\mathrm{VZV})$, rubeola, measles, JC virus, brucellosis, syphilis, mycoplasma, legionella, and Lyme. A human herpesvirus (HHV-6) test was not performed. However, a cytomegalovirus (CMV) reactivation was detected through serological CMV studies (detectable IgM CMV anti-bodies and a high level of IgG CMV anti-bodies), confirmed later using blood CMV DNA count by PCR.

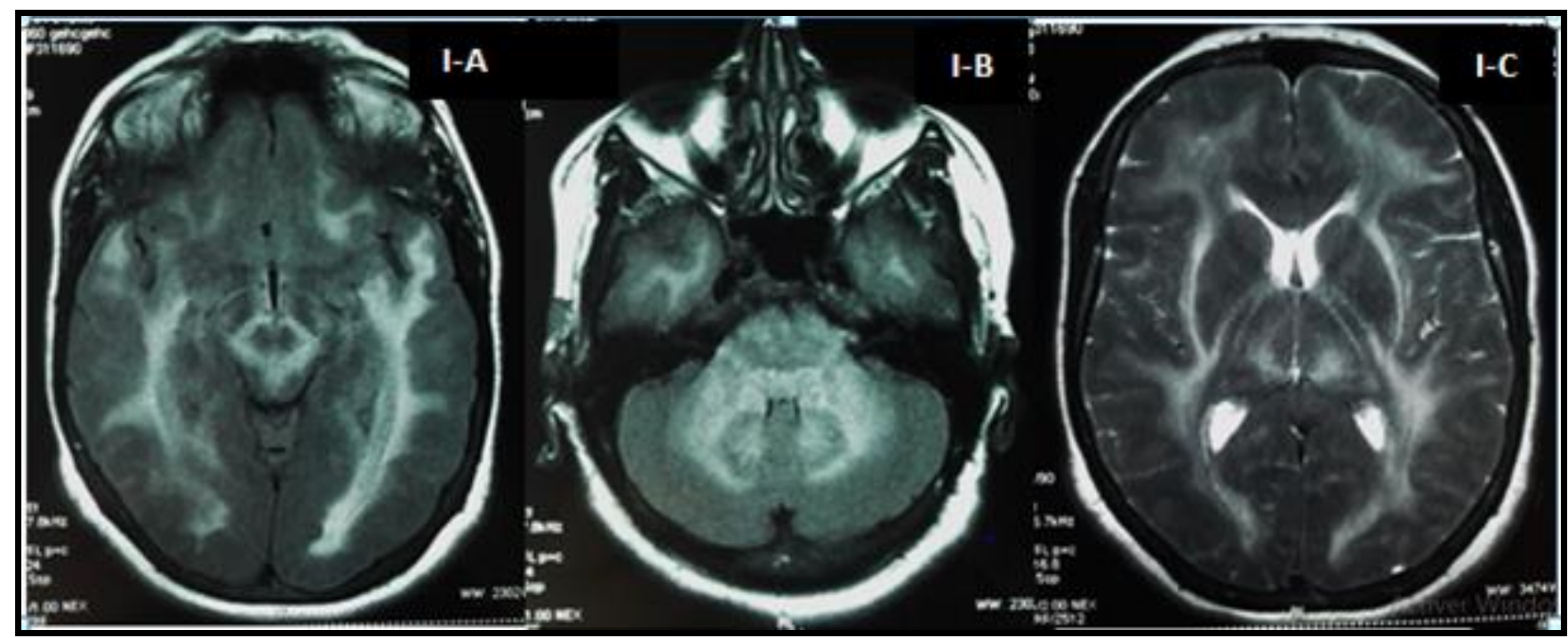

Figure I: Axial T2-weighted and FLAIR-weighted sequences of Brain MRI at onset showing bilateral, diffuse hyper-T2 lesions in cerebral and cerebellar white matter, the brainstem (I-A), dendate nuclei (I-B) and thalamus nuclei (I-C).

The clinical presentation strongly suggested a diagnosis of DRESS Subsequently, carbamazepine was discontinued and treatment with intravenous ganciclovir was initiated. Twenty days after carbamazepine withdrawal, the patient's condition remarkably improved: the skin lesions remitted completely, and the fever disappeared. Neurological signs, as well as papillary edema also slowly regressed. Her laboratory tests (lymphocyte count, CRP, and liver enzymes) considerably improved within weeks. On follow-up, two months after recovery, another MRI was performed and showed a significant improvement of cerebral lesions within the brainstem, basal ganglia, and white matter (figure II).

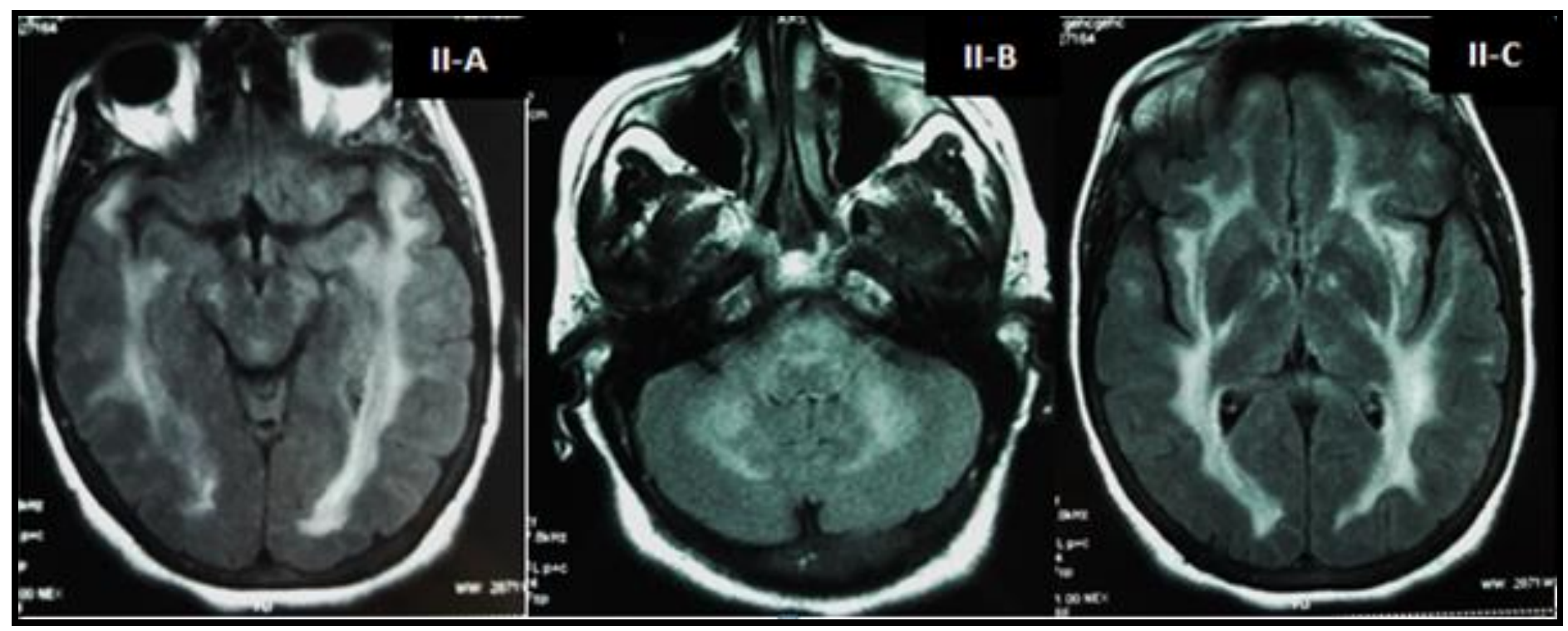

Figure II: Axial FLAIR-weighted sequences of Brain MRI at follow-up showing improvement of lesions in the brainstem (II-A), the cerebellum (II-B) and the thalamus nuclei (II-C). 


\section{Discussion}

DRESS is one of the most severe drug-induced cutaneous adverse reactions and is known for internal organ involvement. It has an estimated overall population risk of between 1 in 1000 and 1 in 10000 drug exposures and has a mortality rate of approximately 10 $\%$ [2]. Several classes of drugs have been reported to be causative agents of DRESS, including anticonvulsants, antibiotics, antiretroviral agents, and analgesics. Specifically, the most commonly implicated drugs are allopurinol, carbamazepine, and sulfasalazine. In this case, carbamazepine appears to be the causative drug for this adverse reaction.

We proceeded to diagnose DRESS for our patient by referring to the Regi SCAR scoring system for classifying DRESS [1] that indicated a "definite" diagnosis. Our patient combined three of four minor criteria consisting of fever, laboratory abnormalities (lymphopenia), and involvement of internal organs (pneumonia and leucoencephalopathy). Additionally, the patient manifested three major criteria necessary for the diagnosis: an acute rash, hospitalization, and the reaction was suspected to be drug related. Moreover, DRESS has a typical late onset between 2 and 6 weeks after drug use [3] and the latency period between drug exposure and the onset of symptoms in our patient was 2 weeks.

Due to a high suspicion of infection when these clinical manifestations occur and unfamiliarity with this rare syndrome, physicians often prescribe unnecessary empirical antibiotic therapy frequently prescribing beta-lactam antibiotics. Such therapies could potentially increase the risk of developing additional drug reactions. In fact, patients with DRESS usually show unexplained crossreactivity to multiple drugs with different structures, including those used after onset [4]. This cross-reactivity to multiple drugs could possibly explain the worsening of our patient's condition after antibiotic administration.

Aside from cutaneous involvement, DRESS is distinguished from other drug reactions by the viral reactivation that characteristically follows the onset of the disease. Its clinical manifestations are highly heterogeneous and follow a variable and unpredictable course beyond the point where the causative drug would be expected to be eliminated from the body [4]. In fact, sequential reactivations of

\section{Conclusion}

We describe an unusual case of a patient with carbamazepineinduced DRESS associated with CMV reactivation that presented as a leuco-encephalopathy. We believe that these neurological manifestations are more likely due to CMV reactivation rather than drug-induced hyper-sensitivity. However, in order to definitively establish the relevance of CMV in this neurological presentation, PCR identification of viral reactivity would be required. various herpes virus have been exclusively demonstrated during the acute stage of DRESS, including human herpesvirus (HHV)-6, HHV-7, Epstein-Barr virus (EBV), and cytomegalovirus (CMV) [5]. In particular, cytomegalovirus (CMV) reactivation normally occurs three to seven weeks after the onset of DRESS that results in uncontrolled viral replication that can lead to fatal diseases including pneumonia, myocarditis, hepatitis, and gastroenteritis [5] Neurological manifestations however are less common during CMV reactivation. One month after carbamazepine, our patient experienced a probable CMV reactivation, although PCR detection of CMV DNA in CSF was not performed. An HHV-6 reactivation was also possible, as most reported cases are commonly associated with HHV-6 reactivation, however; it could not be tested for in this patient.

Recently, several cases were reported describing neurological manifestations during DRESS independent of severe drug reactions or CMV reactivation. These neurologic manifestations included headache, seizure, coma, and motor function impairment that may result from meningitis or encephalitis, peripheral neuropathy, and CNS vasculitis [6].

A review of the literature revealed a few examples of DRESS presenting with leuco-encephalopathy, however; none of them were proven to be associated with CMV reactivation [7]. Although the pathogenesis of leuco-encephalopathy with DRESS is unknown, some authors suggest a key role of HHV-6 in the etiology [8]. For our patient, we propose that reactivation of CMV may have contributed to the development of leuco-encephalopathy as patients with CMV encephalitis often present with rapid onset altered mental status with symptoms of delirium, confusion, apathy, and occasional memory impairment. Furthermore, a brain MRI may show periventricular enhancement on T2 weighted images as was evident in our patient [9]. The improvement of the patient's neurological symptoms and radiological images after anti-viral treatment and the drug withdrawal leads to the hypothesis of CMV reactivation associated with DRESS. Nevertheless, only the detection of CMV DNA in CSF through PCR would confirm such a diagnosis.

Acknowledgments: None. This includes no finding organization, no awards, and no grant recipient.

\section{Declaration:}

$\checkmark$ Funding info: There are no funders to report for this submission.

$\checkmark$ Competing interest: None declared under financial, general, and institutional competing interests.

$\checkmark$ Ethical approval information: The authors attest that the ethical consent for publication of this article was obtained from the patient. 


\section{References}

1. Kardun SH, Sekula P, Veleyrie-Allanore I, Liss Y, Chu CY, et al. (2013) Drug reaction with eosinophilia and systemic symptoms (DRESS): an original multisystem adverse drug reaction. Results from the prospective RegiSCAR study. Br J Dermatol. 169(5): 1071-80.

2. Seitz CS, Pfeuffer P, Raith P, Brocker EB, Trautmann A (2006) Anticonvulsant hypersensitivity syndrome: cross reactivity with tricyclic antidepressant agents. Ann Allergy Asthma Immunol. 97(5): 698-702.

3. Aihara M, Sugita Y, Takahashi S, Nagatani T, Arata S, et al. (2001) Anticonvulsant hypersensitivity syndrome associated with reactivation of cytomegalovirus. British Journal of Dermatology; 144(6): 1231-1234.

4. Shiohara T, Mizukawa Y (2019) Drug-induced hypersensitivity syndrome (DiHS)/drug reaction with eosinophilia and systemic symptoms (DRESS) : An update in 2019. Allergol Int. 68(3): 301-308.
5. Mizukawa Y, Hirahara K, Kano Y, Shiohara T (2019) Druginduced hypersensitivity syndrome/drug reaction with eosinophilia and systemic symptoms severity score: A useful tool for assessing disease severity and predicting fatal cytomegalovirus disease. Jam Acad Dermatol. 80(3): 670-678.

6. Mesec A, Rot U, Perkovic T, Lunder T, Sibanc B (1999) Carbamazepine hypersensitivity syndrome presenting as vasculitis of the CNS. Case conference. 66(2): 249-250.

7. Yang J, Wang G, Fu M (2009) Drug reaction with eosinophilia and systemic symptoms presenting as leukoencephalopathy. Journal of Dermatology. 46(8): e305-e307.

8. Suthar R, Sankhyan N, Shree H, Singhi P (2017) Reversible vegetative state in a child due to drug reaction with eosinophilia and systemic symptoms. Indian J Pediatr. 84(3): 249-250.

9. McCutchan JA (1995) Cytomegalovirus infections of the nervous system in patients with AIDS. Clin Infect Dis. 20(4): $747-54$. 